\title{
Comparing Ranque tubes of circular and square cross section
}

\author{
Ivan K. Kabardin ${ }^{1, *}$, Vladimir G. Meledin ${ }^{1}$, Nikolay I. Yavorsky ${ }^{2}$, Vladimir A. Pavlov ${ }^{1}$, \\ Margarita Kh. Pravdina ${ }^{1}$, Dmitry $V$. Kulikov ${ }^{1}$, and Valentina I. Polyakova ${ }^{1}$ \\ ${ }^{1}$ Kutateladze Institute of Thermophysics SB RAS, 630090, 1 Academician Lavrentiev Avenue, \\ Novosibirsk, 630090, Russia. \\ ${ }^{2}$ Novosibirsk State University, 2 Pirogova Str., Novosibirsk, 630090, Russia.
}

\begin{abstract}
The efficiency of temperature end energy separation is compared for Ranque tubes of circular and square cross section, with the square side equal to the circle diameter. The "square" tube demonstrates approximately two times less efficiency, yet the separation effect still being evidently presented.
\end{abstract}

Experimental study of the vortex effect efficiency in Ranque-Hilsh tubes is presented. The insufficience of the available experimental data and theoretical approaches to the understanding of processes inside a vortex tube are well-known [1]. Systematic and detailed experiments are now strongly needed for verification of theoretical models, the techniques being preferable that do not or just slightly disturb the flow. So, the use of optical diagnostic has allowed a considerable progress in the study of inside swirling flow [2-5]. In [2] by means of Gilbert optics the existence of a large-scale vortex structure in the form of a double spiral in the field of optical phase density was for the first time diagnosed. The spiral arises on a plane end cover at the hot exit and propagates along the axis in the cold exit direction. In [3-4] an attempt was made to diagnose this structure by means of LDA. The sufficient nonstationarity of temperature and velocity was showed. While in [5] a circular tube is investigated, in [2-4] the tube was studied with square cross section all along for the convenience and delicacy of optical diagnostic. It was found that in spite of such a modernization the Ranque effect is distinctly manifested though the tube does not show any record features.

At this stage, the authors proceed to investigate the velocity fields inside the same tube by means of optical field diagnostic - PIV [6]. At that the first step is a detailed investigation of tube efficiency in a wide region of regime parameters for the square tube and comparison of results with a circular tube with the diameter defined as that of inscribed circle.

It should be noted that a significant amount of new publications has appeared in recent times with detailed description of certain vortex tube designs and corresponding efficiencies, such as, for example, [7-8]. The data is supposed to help not only to optimize the technical use but also to verify theoretical models. In our case the detailed data is needed as a road map guiding the field measurements inside the tube.

*Corresponding author: kabardin@itp.nsc.ru 
Two variants of investigated vortex tubes are shown in Figure 1. The tubes include the two-slit swirl apparatus, the accelerating vortex chamber, the working channel, the diaphragm with connected cylinder channel at the cold exit and the radial diffuser at the hot exit. The hot exit is encased in a buffer volume that may be opened to atmosphere or have an exit to it regulated by the valve.

The compressed air enters the vortex chamber through the two tangential slits of 40 $\mathrm{mm}^{2}$ square each at the radii $R 1=65 \mathrm{~mm}$. The opened top cover of the vortex chamber is hyperbolic-shaped that keeps the velocity circulation almost constant up to the entrance into the working channel of the tube at $R 2=17 \mathrm{~mm}$. Thus, the tangential velocity at the entrance of the tube is enlarged as compared to the slit exit velocity. The channel length is $450 \mathrm{~mm}$. The circular tube is made of duralumin. Thermo insulation is not involved. The sections of square tube are made of duralumin with two opposite optical glass windows in the measurement part.

The radial diffuser at the hot exit of the channel may be used as a baffler, the precise opening from 0.5 to $1.5 \mathrm{~mm}$ being adjusted to regulate the cold fraction that is the ratio of cold flow rate to the inlet one $-\mu=G_{c} / G_{i n}$. The parameter $\mu$ could also be regulated by the hot exit valve at maximum opening of $1.5 \mathrm{~mm}$ between disks.

At the cold exit, there is a diaphragm with adjusted tube of $10 \mathrm{~mm}$ diameter. The square side and the circle diameter in cross sections are of $34 \mathrm{~mm}$.

The flow rates at the entrance and the exits were measured by orifice flowmeters each calibrated by a standard flowmeter PC-4 Ultra from a company "IRVIS", with the error not exceeding $0.5 \%$. The temperature and pressure values were measured by the probes as follows: DS18B20 temperature sensors $(223 \ldots 398 \mathrm{~K}$, error less than $0.1 \mathrm{~K})$, pressure sensors SDV-I-2,50-1,60-1,00-4- $20 \mathrm{~mA}$ (0-10 bar, 0.1\% accuracy), pressure drop sensors Honeywell MPX50100 (0-1 bar, $0.1 \%$ accuracy) and SDV-D-2,50-1,60-1,00-4- $20 \mathrm{~mA}$ (010 bar, $0.1 \%$ accuracy).

The cold fraction $\mu$, ranged from 0.2 to 0.9 . The incoming air overpressure $P$ changed from 1 to 7 Bar. The income flow rate $G_{\text {in }}$ came up to $0.07 \mathrm{~kg} / \mathrm{s}$.
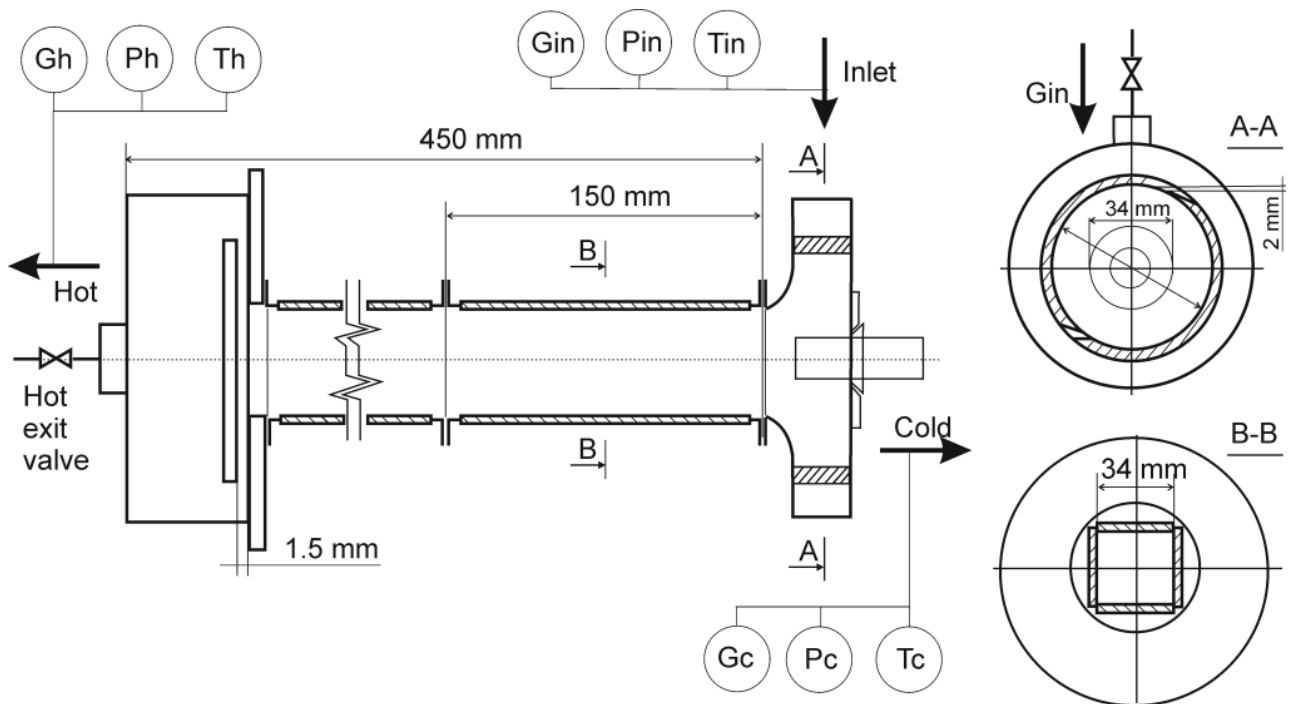

Fig.1. The draft of setup

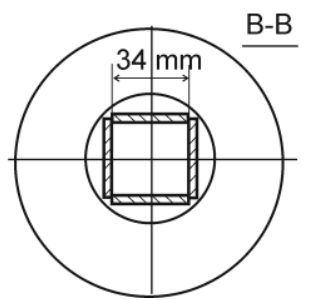



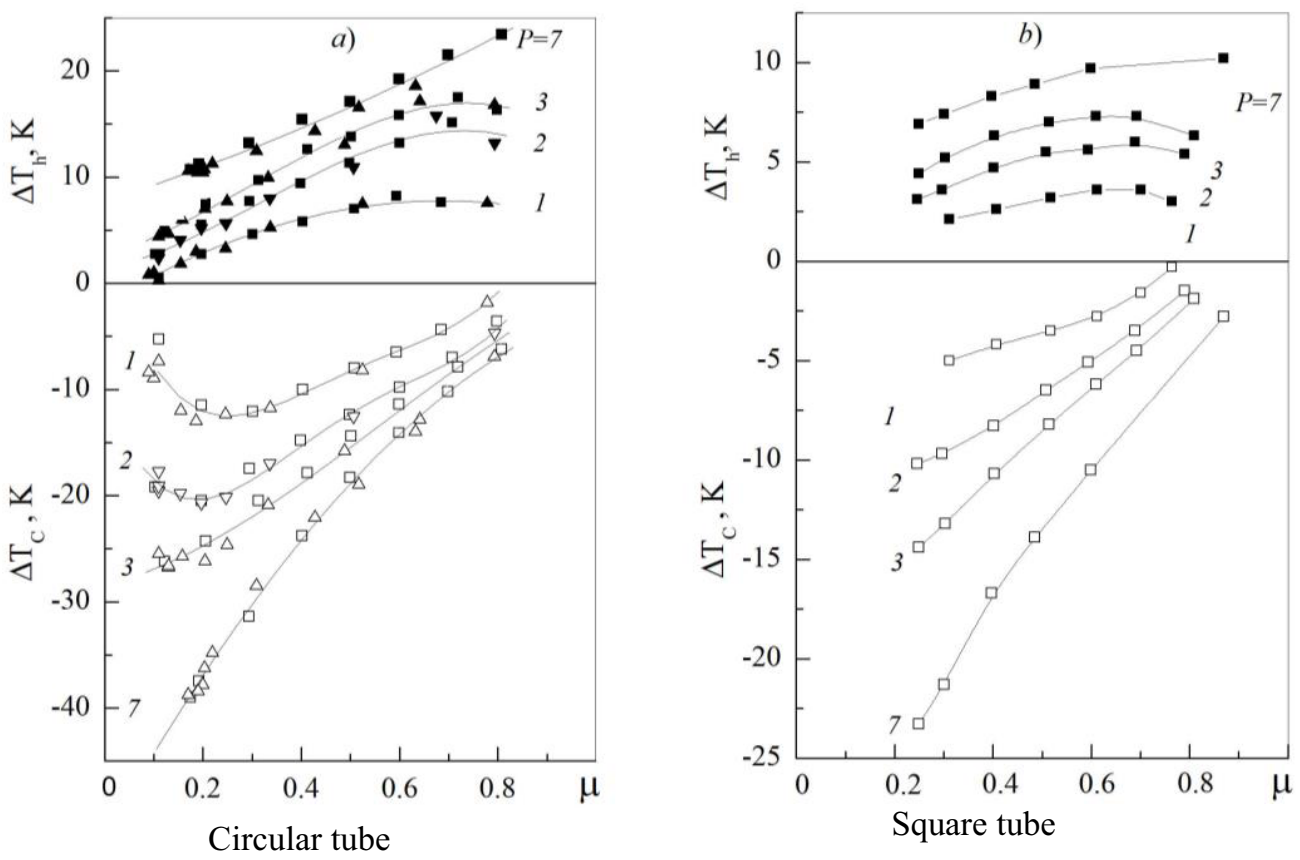

Fig. 2. Temperature separation.

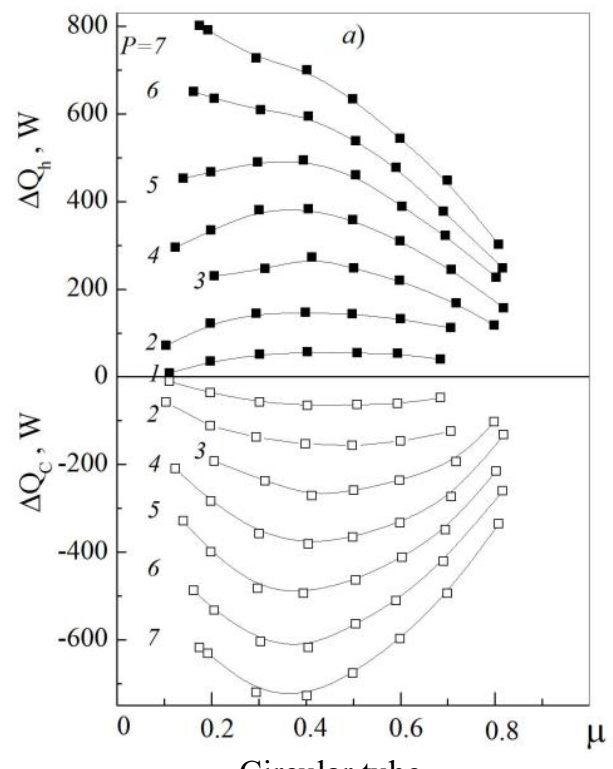

Circular tube

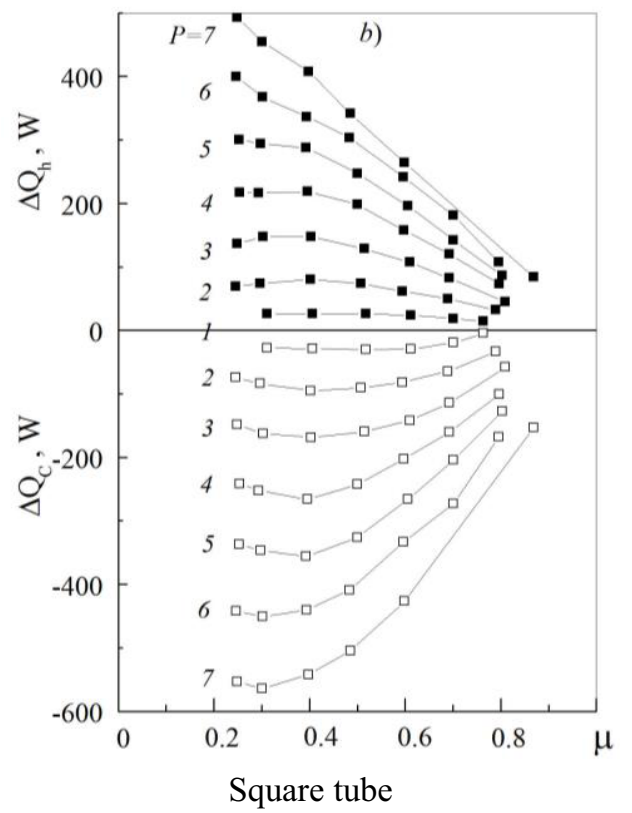

Fig. 3. Energy separation.

The detailed regime maps are executed for circular and square Ranque tubes presenting temperature and energy separation. Figure 2a) shows the circular tube temperature separation data with square points, obtained by regulation of $\mu$ by the valve, and with the triangle points, obtained by varying the opening of the radial diffuser at the hot exit. The 
data show no difference in regulation means. Figures near curves denote the incoming air overpressure (Bar).

In Figure 2b) the experiments on the square tube are presented with only valve regulation of $\mu$. Figures 3a) and 3b) present the energy separation for circular and square tubes with valve regulation of $\mu$. The value of $\Delta Q$ (Watts) is obtained multiplying of $\Delta T$, corresponding mass flow rate and specific thermal capacity.

The comparison of the circular and square tubes show almost equal mass flow rate at the same inlet pressure though the temperature separation in the circular tube is about twice as large as that in the square one. For reference the work [9] should be noted where confined swirling flows in the closed polygonal and circular containers were studied. The reduction of temperature separation in the square tube as compared to the circular one with almost no change in hydrodynamic resistance needs a detailed explanation. Yet the amount of separation in the square tube is still evidently presented, that makes it possible and useful to analyze the Ranque effect by aforementioned optical diagnostics. The detailed data on separation features will make a road map guiding the field measurements inside the tube.

The work is financially supported by RFBR-16-08-01120 A and RFBR 15-08-00186 A.

\section{References}

1. Y. Xue, M. Arjomandi, R. Kelso, Exp. Thermal Fluid Science 34, 8 (2010).

2. V.A. Arbuzov, Y.N. Dubnishchev, A.V. Lebedev, M.K. Pravdina, N.I. Yavorsky, Tech. Phys. Lett. 23, 23 (1997).

3. Y.N. Dubnishchev, V.G. Meledin, V.A. Pavlov, N.I. Yavorsky, Thermophys. Aeromech. 10, 4 (2003) .

4. I.K. Kabardin, V.G. Meledin, N.I. Yavorsky, V.A. Pavlov, M.H. Pravdina, D.V. Kulikov, V.V. Rahmanov, ICMAR AIP Conference Proceeding 1770 (2016).

5. U. Doll, M. Beversdorff, G. Stockhausen, C. Willert, D. Schlüß, C. Morsbach, 17th Int. Symp. on Appl. of Laser Techniques to Fluid Mechanics. Lisbon, Portugal (2014).

6. M. Raffel, C.E. Willert, J. Kompenhans, Particle Imaging Velocimetry (Berlin: Springer-Verlag, 2001).

7. S.N. Jadhav, V.D. Wagh, M.N. Patil, B.V. Kawale, P.P. Patunkar., Int. J. Technology Enchancments Emerging Eng. Res. 3, 4 (2015).

8. M. Attalla, M. Salem, A. Abo EL-Wafa, Int. J. Mechanical \& Mechatronics Eng. 14, 4 (2014).

9. I.V. Naumov, I.Yu. Podolskaya, J. Fluid Mech. (2017) (to be published). 